\title{
XLV. Osmotic pressure from the standpoint of the kinetic theory
}

\section{T. Martin Lowry D.Sc.}

To cite this article: T. Martin Lowry D.Sc. (1907) XLV. Osmotic pressure from the standpoint of the kinetic theory, Philosophical Magazine Series 6, 13:76, 552-559, DOI: 10.1080/14786440709463630

To link to this article: http://dx.doi.org/10.1080/14786440709463630

曲 Published online: 16 Apr 2009.

Submit your article to this journal $\pi$

Џ Article views: 5

Q View related articles $₫$ 


\section{[ 552$]$}

XLV. Osmotic Pressure from the Standpoint of the Kinetic
Theory. By T. MARTIN LowRy, D.Sc.*

van't Hoff's Gas-Theory of Osmotic Pressure.

T $N$ any discussion of the origin and mechanism of osmotic pressure it is necessary to take into account, as a factor of fundamental importance, the fact-now fully established by experiment + - that the osmotic pressure of a dilute solution can be calculated with a very close approach to accuracy by means of the formula $\mathrm{PV}=\mathrm{RT}$, which expresses the relationship between the pressure and volume of a gas. Not only is the osmotic pressure proportional to the concentration of the solution and to its absolute temperature, but the constant $R$ has the same value in the two cases, so that the osmotic pressure is actually identical in magnitude with the pressure exerted by a gas of equal molecular concentration. This result could be predicted on general grounds in the case of gaseous osmotic pressures (Arrhenius, Zeit. phys. Chem. 1889, iii. p. 119; Ramsay, Phil. Mag. 1894, xxxviii. p. 206); but the conditions prevailing in a liquid are so far different that the gas-analogy, though suggestive, would be utterly insufficient to justify the application of the gas-laws to osmotic pressure unless these could be verified experimentally or established on an independent theoretical basis.

When, however, the discovery was made by van't Hoff (Zeit.plys. Chem. 1887, i.pp. 481-508) that osmotic pressure in liquids is actually identical in magnitude with the pressure of a gas exposed to similar conditions of temperature and pressure, it was generally assumed that the two phenomena must be essentially similar in character, and that the osmotic pressure produced by dissolving sugar in water was due, as in gases, to the bombardment of semi-permeable membrane by the molecules of the solute (van't Hoff', loc. cit. p. 482 ; B. A. Report, 1890, p. 336; Zeit.phys. Chem. 1890, v. pp. 174-176). Van 't Hoff's theory had the merit of giving a simple quantitative explanation of osmotic pressure, and was not unreasonable when applied to the osmotic pressure of gases dissolved in liquid (as well as in gaseous) solvents. The conception was, however, not easy to apply to the more ordinary cases of osmotic pressure, such as those afforded by aqueous sugar solutions. In most cases the solute molecules have a very

* Communicated by the Faraday Society (Abstract of a contribution to a general discussion on "Osmotic Pressure" held on Jan. 29, 1907).

$\dagger$ Pfeffer, Osmotische Untersuchungen, Leipzig, 1877; Morse \& Fraser, Amer. Chem. Journ. 1905, xxxiv. p. 1 (1906); compare also the experiments of Griffiths on the freezing-points of dilute aqueous solutions. 
slight mobility, and in the absence of the solvent are unable to produce any hydrostatic pressure whatever upon the walls of the containing vessel. It is therefore scarcely reasonable to attribute the whole action of the solution to the relatively inert solute, whilst neglecting the very active part played by the solvent (Fitzgerald, B. A. Report, 1890, p. 327).

\section{The Mechanism of Osmotic Pressure.}

There can at the present time be no doubt that osmotic pressure depends essentially on the phenomena of selective solubility. The palladium membrane used by Ramsay. to develop a gaseous osmotic pressure acts in virtue of its ability to absorb or dissolve hydrogen, but not nitrogen ; the amount of hydrogen absorbed depends on the pressure, and equilibrium. is attained when the partial pressure of the hydrogen inside the vessil is equal to its total pressure outside. A watermembrane has been used by Nernst to develop an osmotic pressure between ether and an ethereal solution of benzene, the former being soluble and the latter insoluble in water. Copper ferrocyanide, which absorbs or dissolves water and certain salts but not sugars, forms an efficient semi-permeable membrane for aqueous sugar solutions, but not for salt solutions. The presence of the sugar diminishes the solubility of the water in the membrane, and a flow of liquid is set up because the membrane when saturated with regard to the water on one side is supersaturated with regard to the solution on the other side. The amount of water taken up by the membrane can, however, be increased by compressing the liquid, and it is thus possible to counterbalance the decrease of solubility due to the sugar, and by equalizing the solubility on the two sides of the membrane to stop the flow of solvent into the solution. The pressure required to equalize the solubilities and stop the flow is the so-called "osmotic pressure," and it may again be urged that there is no a priori reason for supposing that this would be the same as that produced by a gas of equal molecular concentration.

Pickering's theory (Ber. 1891, xxiv. p. 3639) that the action of the semi-permeable membrane depends on the relative size of the molecules, has not been confirmed. The similar mechanical theory of Sutherland (Phil. Mag. 1897 [5] xliv. pp. 493-498), that the membrume consists of "meshes" through which water but not sugar can pass appears to be equally untenable and need not be discussed here. The theories of Traube and of Armstrong are referred to later. 


\section{Poynting's Theory.}

Much interest was aroused by the publication in 1896 of a paper by Professor Poynting (Phil. Mag. [5] xlii. pp. 289-301) in which an explanation was given of the osmotic pressure of liquids which afforded an alternative to van't Hoff's gastheory, and had the advantage that the active part in osmosis was assigned to the solvent rather than to the solute. In many respects the view he advocated is very similar to that described below, but the two theories differ in one essential point, and consequently lead to widely different explanations of the origin of osmotic pressure. In particular, Poynting was led to assume that osmotic pressure was due to the formation of labile hydrates; and it became necessary, as Whetham pointed out (Nature, Oct. 15, 1896), to assume that all substances which gave a normal osmotic pressure were monohydrated, whilst the double osmotic pressure of binary salts might be ascribed to the formation of a dihydrate or of two monohydrated ions *. The invariable formation of loose monohydrates was so improbable, and is so far in contradiction to recent work on the hydrates present in solution, that Poynting's theory has failed to secure general acceptance as an explanation of the osmotic phenomena.

\section{Osmotic Pressure as a Kinetic Phenomenon.}

The theory of osmotic pressure now described formed the subject of a paper read before the Chemical Society of the Central Technical College as long ago as May 1896; but it was only recently that it was recognized as being sufficiently novel to warrant further publicity.

The starting-point of the theory is a consideration of the conditions prevailing at the surface of separation of the solution and the semi-permeable membrane, which may be either a layer of copper ferrocyanide or merely the boundary between liquid and vapour or liquid and ice. The simplest of these cases is undoubtedly that which involves the equilibrium between liquid and vapour. In this case the kinetic theory postulates a continual process of evaporation, whereby rapidly moving particles are constantly escaping from the surface of the liquid into the vapour space. This is balanced by the condensation of practically all the molecules of the vapour that impinge on the liquid surface. When the vapour reaches a certain concentration the rate of condensation becomes equal to the rate of evaporation, and a condition of equilibrium is attained, not because evaporation has

* Compare I. Traube, Ann. Phys. Chem. 1897, ii. 62. pp. 490-506. 
ceased, but because it is neutralized by an equal and opposite process of condensation. In the case of a non-volatile liquid the mobility of the relatively heavy molecules or molecular complexes is so small that very few are able to escape, and the maximum vapour-pressure is inappreciable.

If now a solution be prepared by mixing a volatile and a non-volatile liquid, or by dissolving a non-volatile solid in a volatile liquid, the surface will contain both kinds of molecules. If one of the solvent molecules be struck by a rapidly moving molecule from the interior of the liquid, it will be projected into the vapour space. If, however, one of the non-volatile molecules be struck, it will be unable to escape, and the solvent particle will rebound in much the same way as if it had struck the wall of the containing vessel. The rate of evaporation is therefore reduced by the addition to the solvent of a non-volatile solute. On the other hand, it is probable that the presence of the non-volatile molecules would not interfere with the rate of condensation of the vapour. This point is of fundamental importance, as the opposite view was advocated by Poynting, who supposed that condensation and evaporation would be checked to an equal extent, just as if the surface had been covered by a plate of perforated zinc. It must be remembered, however, that a considerable upward velocity is required before a molecule can escape from the surface of a liquid, and that a molecule descending with even the smallest downward velocity wenld have little chance of escaping when once it came within the range of attraction of the liquid. Even if, on reaching the liquid surface, a vapour molecule should strike against a nonvolatile molecule of the solute, the attraction of the neighbouring molecules of the solvent would be sufficient to hold it, and thus ensure its condensation.

It need scarcely be pointed out that similar conditions would prevail at the surface separating liquid and ice, or at the surface of one of the more conventional semi-permeable membranes. In the former case the presence of a nonisomorphous solute might prevent the adhesion to the ice of a molecule of solvent moving towards it but separated from it by a molecule of solute. On the other hand, it would not prevent the melting off or dissolution of an ice molecule if the average kinetic energy ( $i$. e. temperature) of the ice were raised by the latent heat of crystallization of other molecules passing from the liquid to the solid state. In the case of a membrane such as copper ferrocyanide the solute would check the escape of solvent molecules from the solution into the membrane, but would not oppose the return of wanderers 
migrating from the membrane back into the solution. This would lead to a disturbance of the equilibrium between the solvent and the liquids on either side, and would be sufficient to produce an osmotic flow and a consequent osmotic pressure.

\section{Deduction of the Gas-Formula from the Kinetic Theory of Osmotic Pressure.}

Of the theories of osmotic pressure that have hitherto been put forward, that of van 't Hoff is the one that leads most directly to a simple quantitative explanation of the phenomena. Poynting, in developing his theory quantitatively, considered that solute molecules and stable compounds of solvent and solute would merely alter the effective surface of the liquid without changing the relative rates. of evaporation and condensation ; only in the case of labile compounds was it considered possible that the rate of evaporation might be checked without altering the rate of condensation; on this basis a quantitative explanation was only possible on the impracticable assumption that all ordinary solutes are loosely monohydrated in aqueous solutions. The more recent theory that osmotic pressure depends on a disturbance in the equilibrium between simple and polymerized solvent molecules, e. g., $n \mathrm{H}_{2} \mathrm{O} \rightleftarrows \mathrm{H}_{2 n} \mathrm{O}_{n}$ (Armstrong, Proc. Roy. Soc. 1906, A. lxxviii. pp. 264-271), has also, as yet, failed to yield a quantitative interpretation of the pressures produced.

The quantitative interpretation of the kinetic theory of osmotic pressure follows at once from Nernst's proof of the relationship between osmotic pressure and vapour pressure ('Theoretical Chemistry,'pp. 124-129). Thus if $N$ be the number of gram-molecules of solvent, and $n$ the number of gram-molecules of solute in unit volume of the solution, the validity of the gas-formula for osmotic pressure is readily deducible from the equation

$$
\frac{\mathrm{N}}{\mathrm{N}+n}=\frac{p^{\prime}}{p},
$$

where $p$ is the vapour-pressure of the solvent, and $p^{\prime}$ that of the solution*. If the simple assumption is made that the molecules of solvent are uniformly distributed in the surface layer, and that the spacing or packing is the same as in the pure solvent, it follows at once that the rates of evaporation from unit surface of solvent and solution will be in the ratio

* The value of $\mathrm{N}$ is determined by the molecular weight of the solvent as it exists in the vapour, and not by its molecular weight in the liquid state. 
$\mathrm{N}+n$ to $\mathrm{N}$, and that this will also be the ratio of the vapourpressures, as required by the above formula. The argument is, of course, identical with that used by Poynting to show that each molecule of solute must destroy the mobility of a molecule of solvent; but, whereas he was led to assume the regular formation of labile monohydrates, the theory given above merely postulates that the mobility of a solvent molecule is destroyed when its place in the surface of the liquid is occupied by a moleeule of the solute.

\section{Surface Structure of Liquids.}

It will at once be noticed that in its simplest form the kinetic theory of osmotic pressure would indicate that the pressures calculated from the gas-formula might be subject to a small correction for the volume changes accompanying dissolution. Whether such corrections are necessary can only be determined by experiment, but the evidence now available points to a very close agreement between the values observed and calculated for dilute solutions. If this identity should be confirmed, it will be possible to deduce from observations of osmotic pressure some information in reference to the surface structure of liqurds, since if the agreement is exact there must be an equally exact replacement of solvent by solute in the surface of the liquid. Thus in view of the different masses of the molecules that may be dissolved in the same solute and yield identical osmotic pressures, there must be a considerable spacing between the actual molecules in the surface. Again, it may be noted that this exact replacement does not take place in the interior of the liquid where the molecular volumes of different solutes differ widely, and when calculated in the conventional way may even have a negative value. It is, however, by no means improbable that the marsballing of the molecules on the frontiers of the liquid may be governed by a stricter discipline than that which prevails in the interior, and that the surface molecules may even be forced to conform to the exact regulations which govern the replacement of molecules in solid solutions or isomorphous mixtures.

\section{Formation of Complexes.}

The formation in the solution of loose complexes of solvent with solute or of solvent molecules with one another has not yet been referred to, but presents no difficulty in the development of the theory. Such complexes are usually formed without any large change of volume, and under the stricter 
conditions prevailing at the surface no alteration in the area of surface occupied need result from the linking up of the "residual affinities" of the molecules. Neither need it be supposed that the rate of evaporation would be affected otherwise than by a general reduction of mobility * due to the chemical attraction of the molecules and producing equal effects in solvent and in dilute solution. Thus, as there is no change of energy involved in the replacement of one solvent molecule in a complex by another from outside, a rapidly moving particle impinging on a complex might drive a conbined solvent molecule into the vapour space and itself occupy the vacant position in the complex, the effects produced being much the same as if no complex existed. A similar statement would apply to molecules of solvent attached to the solute in the form of labile hydrates or compounds; a free solvent molecule impinging on a combined molecule in the surface of the liquid might drive it out and take its place; but if it should impinge on the nuclear solute molecule it would be repulsed and driven back into the interior, just as it would be by an uncombined molecule of solute.

\section{Concentrated Solutions.}

No attempt has been made in the above to account quantitatively for the osmotic pressure of concentrated solutions. Even in the case of gases, the equation PV=RT only applies strictly to a material gas within narrow limits of pressure; but Morse and Fraser's experiments indicate a much wider applicability when the formula is applied to osmotic pressures, provided only that for concentrated solutions $\mathrm{V}$ is interpreted as the volume of solvent used to dissolve the solute and not the total volume of the solution. It need scarcely be pointed out that this modification is very similar in type to the co-volume correction in van der Waals's equation.

\section{Osmotic Pressure and Surface Tension.}

In view of the close relationship that has been indicated between osmotic pressure and surface structure it would not be surprising that a relationship should exist between surface tension and osu otic pressure. Such a relationship bas been postulated theoretically by Truube, who supposes that osmotic pressure depends on a tendency to equalize the surface tension of the two liquids, and has been confirned experimentally by

* Compare the reduction of mobility caused by liquid cohesion in a gras and represented by the quantity $\frac{a}{v^{2}}$ in van der Waals's equation. 
Batelli and Stephanini (Atti R. Accad. Lincei, 1905, (v.) 14. ii. pp. 3-14), who find that solutions of equal surface tension have equal osmotic pressures, even in cases in which the solutions are not nominally equimolecular.

In conclusion it may be pointed out that whilst the views advocated above were comparatively novel ten years ago, the idea that osmotic pressure depends on the activity of the solvent rather than on that of the solute has now become widely accepted, and has been advocated not only by Poynting, but also by Armstrong (Enc. Brit. xxvi. p. 739), Beillby (B. A. Report, South Africa, 1905, p. 301), and others. At the present time, therefore, the only points for which any degree of novelty can be claimed are in reference to the mechanism by which the activity of the solvent at the surface of the liquid is reduced by the "blocking action" of the solute operating in one direction only, and to the possibility of deducing from the osmotic phenomena information as to the surface structure of liquids.

130 Horseferry Road, Westminster, S.W.

XIVI. Notices respecting New Books.

Les Ultramicroscopes et les Objets Ultramicroscopiques. Par A. Cотток et H. Mouton. Paris : Masson \& Cie. 1906. Pp. 232.

THE important part played by the microscope in almost every department of science, pure and applied, readily accounts for the large amount of attention which this instrument has received and for the rapid improvements in its construction-improvements which have rendered it one of the most perfect instruments at our disposal. The limits beyond which a microscope of the ordinary type ceases to be serviceable-limits imposed on it by diffraction phenomena-having been reached, investigators began to turn their attention to means whereby objects too tiny to be visible under ordinary conditions could be so rendered, by the use of special devices, although such devices do not enable us to study the exact form or minute structure of the object viewed.

An account of the most recent researches in this direction will be found in Messrs. Cotton and Mouton's excellent little treatise, excellent alike from the point of view of the selection and arrangement of subject-matter, and that of simplicity of treatmente The subject is introduced by a very clear sketch of the physical theory of the microscope and the limits of visibility. The use of radiations of short wave-length is then dealt with, and in the next chapter is given an account of the conditions under which ultramicroscopic objects may be rendered visible, and a description of the special instruments devised by Siedentopf and Zsigmondy, and 